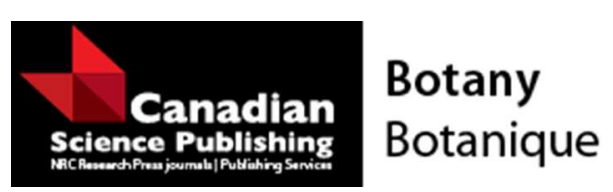

\title{
The Arabidopsis PARAQUAT RESISTANT1 mutant accumulates leucine upon dark treatment.
}

\begin{tabular}{|r|l|}
\hline Journal: & Botany \\
\hline Manuscript ID & cjb-2016-0321.R1 \\
\hline Manuscript Type: & Article \\
\hline Date Submitted by the Author: & 24-Feb-2017 \\
\hline $\begin{array}{r}\text { Complete List of Authors: } \\
\text { Please Select from this Special } \\
\text { Issues list if applicable: }\end{array}$ & $\begin{array}{l}\text { Of Alberta, Agriculture and Forestry } \\
\text { Good, Allen; University of Alberta }\end{array}$ \\
\hline Keyword: & LAT4, PAR1, L-type amino acid transporter, amino acid, transporter \\
\hline & \\
\hline
\end{tabular}

\section{SCHOLARONE ${ }^{\text {TW }}$ \\ Manuscripts}


Title: The Arabidopsis PARAQUAT RESISTANT1 mutant accumulates leucine upon dark treatment.

\section{Authors:}

1. Rowshon A. Begam ${ }^{1 *}$

Department of Biological Sciences, University of Alberta, Edmonton, AB, T6G 2E9, Canada. E-mail: begam@ualberta.ca

2. Allen G. Good

5-180 CCIS, Department of Biological Sciences, University of Alberta, Edmonton, AB, T6G 2E9, Canada. E-mail: allen.good@ualberta.ca

*Corresponding author

Rowshon A. Begam

Alberta Legislature Annex, $5^{\text {th }}$ floor

9718-107 St NW, Edmonton, AB, Canada, T5K 2N6,

Phone: 7804222070

Fax: 7804275921

${ }^{1}$ Current affiliation: Department of Agriculture and Forestry, Government of Alberta, Legislature Annex, $5^{\text {th }}$ fl., 9718-107 St NW, Edmonton, AB, Canada, T5K 2N6, E-mail: rowshon.begam@gov.ab.ca 


\begin{abstract}
The Arabidopsis PARAQUAT RESISTANT1 (PAR1) was classified as L-TYPE AMINO ACID TRANSPORTER 4 (LAT4) based on a phylogenetic analysis of selected genes from Saccharomyces cerevisiae, Arabidopsis thaliana, and Homo sapiens that clustered LAT4 with four other members as a LAT family in Arabidopsis. In-silico analysis of the Arabidopsis LATs identified an amino acid permease domain and motifs that are common in amino acid transporters. However, their role in amino acid transport remained to be studied. A knockout mutant for PAR1/LAT4 gene, reported here as par1-5, showed significantly altered growth compared to wild type on leucine-containing growth medium. Mutant par1-5 seedlings showed reduced biomass compared to wild type on nitrate-containing Murashige and Skoog growth medium which was further reduced when grown on medium containing nitrate and leucine. Radio-labeled leucine uptake studies using leaf protoplasts and seedlings showed increased accumulation of leucine in par1-5 mutants compared to wild type. Increased accumulation of leucine in par1-5 was detected when seedlings or protoplasts were treated in the dark prior to isotopic feeding. These studies suggest that the PAR1/LAT4 protein, in addition to its ability to mediate paraquat and polyamine transport, possess leucine transport activity that is regulated by physiological conditions such as dark induction.
\end{abstract}

Keywords: amino acid; transporter, LAT4; L-type amino acid transporter; PAR1 


\section{Introduction}

Plants take up nitrogen predominantly in the form of nitrate and ammonium that is assimilated into amino acids through the Glutamine Synthetase (GS)/

Glutamate Synthase (GOGAT) pathway [reviewed in (Lam et al. 1996)]. Some species also take up nitrogen in the form of amino acids and peptides [reviewed in (Nasholm et al. 2009)]. Assimilated nitrogen is transported from the source to sink tissues in the form of amino acids. The transport and distribution of these amino acids requires them to cross plasma and organellar membranes in various organs and tissues. Integral membrane transporter proteins, known as amino acid transporters, mediate the transport of amino acids across membranes. To date, over 60 genes in Arabidopsis thaliana have been annotated as amino acid transporters (Rentsch et al. 2007; Tegeder 2012). A phylogenetic analysis categorized these plant amino acid transporters into three super families: $\underline{\text { Amino }}$

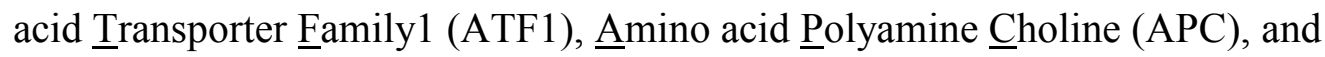

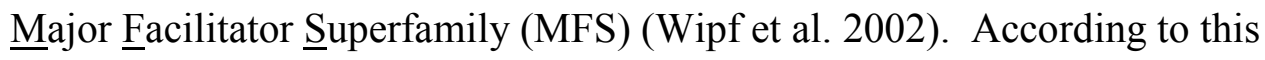
classification, Arabidopsis LATs (LAT1, At5g05630; LAT2, At3g13620; LAT3, At1g31820; LAT4, At1g31830; and LAT5, At3g19553) were classified in the Ltype $\underline{\text { Amino acid }}$ Transporter (LAT) family within the APC superfamily (Wipf et al. 2002). In a later phylogenetic study, these five members of the Arabidopsis LAT family were classified as $\underline{\text { Polyamine }} \underline{\mathrm{H}}^{+}$-Symporters (PHSs) (Okumoto and Pilot 2011). 
Recent experimental studies renamed Arabidopsis LAT1 as Resistant to Methyl Viologen1 (RMV1) and Polyamine Uptake Transporter 3 (PUT3), and LAT4 as

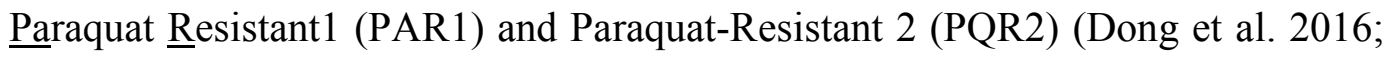
Fujita et al. 2012; Li et al. 2013; Mulangi et al. 2012). In planta studies suggested that Arabidopsis RMV1/LAT1/PUT3 mediates paraquat (a structural analog of polyamine) and cadaverine (a diamine derivative of lysine) import (Fujita et al. 2012; Sagor et al. 2016). Yeast complementation studies showed that this transporter also mediates polyamine and thiamine import (vitamin B1) (Martinis et al. 2016; Mulangi et al. 2012). Arabidopsis PAR1/LAT4/PQR2 was shown to mediate paraquat accumulation into chloroplast, and to have a role in antioxidative stress responses and ABA signaling (Dong et al. 2016; Li et al. 2013).

In this report, through in-silico and in-planta studies, we demonstrate that PAR1/LAT4/PQR2 mediates leucine transport and this activity is regulated by physiological conditions such as dark cycle.

\section{Materials and methods}

In silico analysis of PAR1/LAT4-family proteins

Amino acid sequences of the PAR1/LAT4-family proteins were taken from the plant membrane protein database (http://aramemnon.botanik.unikoeln.de/) and all other protein sequences used in alignment and motif search were taken from the NCBI protein database. Molecular weight and isoelectric point was calculated using Vector NTI 10 (supplementary table S1). Subcellular localization was predicted using AtSubp online prediction tool (Kaundal et al. 2010). InterPro scan was carried out using the InterPro protein sequence analysis and classification 
online tool with the default settings (http://129.175.105.74/genomics/lbmgeiprscan.html). The transmembrane domain prediction was performed using the TMHMM v.2.0 online tool (http://www.cbs.dtu.dk/services/TMHMM/). The cartoon representation of PAR1/LAT4 (Fig. 1a) was produced using an online tool TMRPres2D (http://bioinformatics.biol.uoa.gr/TMRPres2D/). The conserved domain search was performed using the NCBI online conserved domain search tool (http://www.ncbi.nlm.nih.gov/). Amino acid sequence alignment featuring sequence identity, PFAM analysis, and the amino acid permease domain cartoons (Fig. 1b) were produced using the Vector NTI 10 AlignX tool. A motif search in the 26 related proteins including Arabidopsis thaliana, Oryza sativa, Homo sapiens, and Mus musculus LATs (Fig. 1c) was carried out using the online tool Multiple Em for Motif Elicitation (http://meme-suite.org/tools/meme) (Bailey and Elkan 1994), with the default settings except the number of motif which was changed from 3 to 6 . Unknown motifs identified in the LAT proteins were searched against Arabidopsis thaliana, Oryza sativa, Homo sapiens, and Saccharomyces cerevisiae protein databases using online Motif Alignment and Search Tool (http://meme-suite.org/tools/mast) (Bailey and Gribskov 1998). The C-terminal FWC motif search in the Arabidopsis LAT protein sequences was carried out by aligning them with yeast general amino acid permease ScGAP1(CAA82113) sequence using Vector NTI 10 AlignX tool.

Plant growth and analysis 
The Columbia-0 ecotype of Arabidopsis thaliana was used in all studies. All plants (unless otherwise mentioned) were grown on well-aerated fertilized soil in the growth chamber under a $16 / 8$ hours of light/dark regime under $\sim 120 \mu \mathrm{E} \mathrm{m}{ }^{-2}$ $\mathrm{sec}^{-1}$ light intensity at $23^{\circ} \mathrm{C}$ day time temperature. Soil was a commercially available Sunshine Mix formulated with peat moss, coarse perlite, organic nutrient, gypsum, and dolomitic limestone (Terralink Horticulture Inc., Abbotsford, BC, Canada). Plant Prod 20-20-20 fertilizer (Master Plant-Prod Inc., Brampton, ON, Canada) was dissolved in water as per manufacturer's recommended dose and applied to soil during pot preparation.

For phenotypic studies nitrogen-free half-strength MS medium (Murashige and Skoog 1962) was prepared using stock solutions of macronutrients, micronutrients, and vitamin mixture at a molar concentration described in the commercially available MS basal salt mixture (Catalog number ICN2623120, Fisher Scientific, ON, Canada) without ammonium nitrate. To obtain desired nitrogen concentration $(3 \mathrm{mM}$ nitrate and $1 \mathrm{mM}$ or $2 \mathrm{mM}$ leucine, Fig. 2), potassium nitrate and L-leucine (Sigma-Aldrich, Oakville, ON, Canada) were added to the MS-agar medium. For each growth condition, both genotypes were grown on the same plate with 3-5 plate replications. Due to poor growth of the par1-5 seedlings on $2 \mathrm{mM}$ leucine, for each growth condition two seedlings per genotype were weighed per unit sample to minimize technical error. Data analysis and significance tests (two-way t-test) were performed using the software PRISMv.5. The relative percentage of growth in parl-5 seedlings was calculated based on setting the biomass obtained by the corresponding WT at $100 \%$. 


\section{Isolation of the mutant par1-5}

An Arabidopsis line (SALK_119707C) with a T-DNA insertion in the PAR1/LAT4 locus (supplementary figure. S1a) was obtained from the Arabidopsis Biological Resource Centre (Alonso et al. 2003). This line was previously confirmed for being resistant to paraquat, lacking detectable PAR1/LAT4 transcripts, and was named par1-5 (Li et al. 2013). We used the par1-5 line in all studies after confirming by PCR and Reverse Transcriptase (RT)-PCR that it was a homozygous mutant that lacked detectable PAR1/LAT4 transcripts (supplementary figure. S1b). Primers used for RT-PCR were specific to the 5' and 3'-untranslated regions of the PARI/LAT4 locus with sequences as follows: forward 5'-CCGTTTAAAATCTGGTTGACG-3' and reverse 5'GACGAGGGAAATAACTAAG-3'. For RT-PCR, total RNA was extracted, using the RNeasy Plant Mini Kit (Qiagen, Darmstadt, Germany), from 100mg of flash frozen leaf tissues as per manufacturer's instruction. To remove contaminating DNA, RNA was treated using Ambion's DNA-free kit. The quantity and quality of RNA was checked by using Nanodrop and Bioanalyzer (Agilent 2100 Bioanalyzer). One-step RT-PCR using PAR1/LAT4 and Actin2 specific primers in the same reaction vial was performed using SuperScript III One-Step RT-PCR System with Platinum Taq High Fidelity (Catalog No. 12574030). Actin2 was used as a technical control and wild type plant tissues were used as a positive control. The reactions were set up as per manufacturer's instruction and the program for the thermal cycler was as follows: cDNA synthesis at $55^{\circ} \mathrm{C}$ for 30 minutes, pre-denature at $94^{\circ} \mathrm{C}$ for 2 minutes, 40 cycles of: denature at $94^{\circ} \mathrm{C}$ 
for 15 seconds, annealing at $55^{\circ} \mathrm{C}$ for 30 seconds, extension at $68^{\circ} \mathrm{C}$ for 1 minute, final extension at $68^{\circ} \mathrm{C}$ for 5 minutes.

Quantitative RT-PCR for organ specific expression analysis

For the organ specific quantitative RT-PCR (Fig. 3), RNA was extracted from roots, stems, rosette leaves, cauline leaves, senescing leaves, flowers, siliques, and whole plant tissues separately. Total RNA extraction, DNAse treatment, quantification, and quality check was carried out as described above. All samples were adjusted to a final concentration of $50 \mathrm{ng} \cdot \mu \mathrm{l}^{-1}$. For each sample, $2.5 \mu \mathrm{g}$ of total RNA was used in the cDNA synthesis with oligo-dT and random primers (Invitrogen). SuperScript II Reverse Transcriptase (Invitrogen) was used in the reverse transcription reaction for 50 minutes at $42^{\circ} \mathrm{C}$. Elongation Factor $1 \alpha$ (EF1 $\alpha)$ was used as an endogenous control. A TaqMan Gene Expression Assay (assay ID: At02351062_sH for PAR1/LAT4; assay ID: At02337969_g1 for EF1 $\alpha$ ) was used in the PCR amplification with the following program: $95^{\circ} \mathrm{C}$ for 10 minutes followed by 40 cycles of $95^{\circ} \mathrm{C}$ for 15 seconds and $60^{\circ} \mathrm{C}$ for 1 minute. The relative quantitation was calculated using a 7500 Fast System SDS software (Applied Biosystems). Each replication is an average of three reactions and each data point is an average of three biological replications.

In vivo ${ }^{14}$ C-leucine and ${ }^{3} H$-leucine uptake study

Radio-labelled L-leucine was used in all studies. The ${ }^{14} \mathrm{C}$-leucine and ${ }^{3} \mathrm{H}-$ leucine uptake studies were carried out using homozygous par1-5 seedlings compared to wild type as biological control. Three-week old seedlings grown on fertilized peat-based growth medium were used in the uptake studies. For dark treatment, 
seedlings in the growth tray were covered with air-permeable black cover and kept in the dark for 27 hours prior to use. In all studies, a mixture of ${ }^{14} \mathrm{C}$-leucine and ${ }^{3} \mathrm{H}$-leucine was used with the isotopic label in the alpha-carbon and amino group, respectively. Radio-labelled leucine was added to an uptake medium (liquid MS basal salts without sucrose and nitrogen supplement) with cold leucine to a final concentration of $10 \mu \mathrm{M}$. A total of $15 \mathrm{ml}$ uptake medium was used for each replication sample. Seedlings were removed from the uptake medium at twohour time point. Roots were rinsed three times in sterile water for a total of 10 minutes. Roots and shoots were separated immediately after washing and stored directly in glass scintillation vials. Samples were prepared for counting radioactivity using a protocol similar to the one described in (Pratelli et al. 2010). Tissues were dried at $70^{\circ} \mathrm{C}$ overnight and weighed. Samples were digested with $1 \mathrm{ml}$ of $5 \% \mathrm{NaClO}$ overnight at room temperature. Vials were kept open for at least one hour under a fume hood before adding $5 \mathrm{ml}$ Hionic-Fluor (Ultima Gold XR, Perkin Elmer Inc., Waltham, MA, USA) to each vial. Radioactivity in roots and shoots of the same plant were combined to obtain total radioactivity in each sample. Data was presented as leucine concentration in pmol.mg ${ }^{-1} \mathrm{DW} \cdot \mathrm{hr}^{-1}$ (Fig. 4a-d).

To prepare leaf protoplasts, rosette leaves of similar size and age were collected from two-month old plants. For each genotype, 30 leaves were used from 10 plants. Plants were kept in the dark for 24 hours before leaves were collected. Protoplast preparation was performed in the dark using a protocol described by (Yoo et al. 2007). For size analysis, photos of protoplasts were taken using a 
ZEISS Scope A1 light microscope attached to an optronics camera. All photos were taken under the 20x magnification. For each genotype, 100 cells were counted from five photographs from three individual plants. Cell size was measured using PictureFrame (TM) Software Application version 2.3. The program was calibrated using a stage micrometer under the 20x magnification prior to measuring the diameter of the protoplasts. Data was presented as cell size in $\mu \mathrm{m}$. cell $^{-1}$ (Fig. 4e).

For the ${ }^{14} \mathrm{C}$-leucine uptake study, cells of each genotype were counted with a haemocytometer and the final concentration was adjusted to $4 \times 10^{5}$ cells.ml ${ }^{-1}$. The uptake medium was a MMG solution (4mM Mes, 0.4M Mannitol, and $15 \mathrm{mM}$ $\mathrm{MgCl}_{2}$, pH 5.6) (Yoo et al. 2007), with added ${ }^{14} \mathrm{C}$-leucine (618pmol.ml ${ }^{-1}$ ) and cold leucine to a final concentration of $10 \mu \mathrm{M}$. After one hour of uptake under light, cells were rinsed three times with MMG solution before radioactivity was counted in DPM in $4 \times 10^{5}$ cells in each replication vial. Data was presented as leucine concentration in pmol. $10^{5}$ cells.hour ${ }^{-1}$ (Fig.4f)

Free amino acid extraction and HPLC

Free amino acids were extracted using a protocol described in (Tuin and Shelp 1994), from the roots and shoots of WT and parl-5 seedlings, grown under 16/8 light/dark cycle with or without sucrose in the growth medium (Fig. 5a). For the HPLC analysis of free amino acids, freshly weighed samples were ground in 1.5 $\mathrm{ml}$ extraction buffer (methanol: chloroform: water at 65:25:10 with 20 nmol.ml ${ }^{-1}$ internal standard (norvaline and sarcosine) added to each sample. Samples were vortexed and centrifuged to separate the aqueous phase. Chloroform and water 
were added to the aqueous phase at a ratio of $1: 1.5: 5$. Samples were mixed and centrifuged, and the upper aqueous phase was passed through a MILLEX GX $0.22 \mu \mathrm{m}$ filter (UFC30GV0S) followed by a MILLIPORE BIOMAX 5KNMWL MEMBRANE filter (UFV5BCC00). The final filtrate was used in the HPLC analysis using a ZORBAX Rapid Resolution HT Eclipse Plus C18 $1.8 \mu \mathrm{m}$ 3.0x100 mm column. The HPLC analysis was carried out using a protocol described by Agilent Technologies (Woodward et al. 2007). An Agilent Technologies 1200 series automated liquid chromatography system was used to analyze the samples. Samples were derivatized pre-column with OPA (oPhthalaldehyde) and FMOC (9-Fluorenylmethyl chloroformate). All chromatographs were processed in the Agilent Chemstation software suite. The area under the signal peaks was calibrated by the standard calibration curve and normalized by the internal standard norvaline and sarcosine concentration, then all the concentration data were back calculated to the concentration in the samples in nmol.gram ${ }^{-1} \mathrm{FW}$ of shoot. Amino acid concentration in roots was expressed in nmol.2seedlings ${ }^{-1}$ to reflect a relatively accurate concentration of amino acids identified in the root tissues and data for the two genotypes was compared accordingly.

\section{Results}

In-silico characterization of PAR1/LAT4 indicates its role in amino acid transport In Arabidopsis, PAR1/LAT4 is a 495-aa protein with a molecular weight of 54.8 $\mathrm{kDa}$. A secondary structure analysis predicted 10 transmembrane domains in the PAR1/LAT4 protein with both the N- and C-termini in the cytoplasmic side (Fig. 
1a). An InterPro scan identified PAR1/LAT4 as an amino acid/polyamine transporter 1 (IPR002293) family protein containing PF13520 (amino acid permease) and PTHR11785:SF78 (glutamate/GABA antiporter) domains. InterPro analysis predicted its biological process to be amino acid transmembrane transport (GO: 0003333), and its molecular function to be amino acid transmembrane transport activity (GO: 0015171). Similar features were also identified in the other four members of the PAR1/LAT4-family (supplementary table S1). A domain search identified amino acid permease domain PF13520 conserved in all five members of the PAR1/LAT4-family proteins (Fig. 1b). A NCBI blast search against the PDB protein database using BLASTP2.5.1 (Altschul et al. 1997) identified E. coli L-arginine/Agmatine antiporter (AdiC, $\mathrm{pdb} / 5 \mathrm{~J} 4 / \mathrm{A}$ ) as the closest protein with $25 \%$ sequence identity. PAR1/LAT4 shares a number of conserved regions with its family members (supplementary figure S2). A motif search in the PAR1/LAT4 protein along with related proteins including LATs in Arabidopsis, Oryza sativa, Homo sapiens, and Mus musculus identified six common motifs in these proteins (Fig. 1c). Sequences of these motifs were searched again in the Arabidopsis, Oryza sativa, and Homo sapiens protein databases to see which known proteins carry these motifs, and if they can be associated with a common function. These motifs were identified in a number of other proteins including AtCAT1-9, AtBAT1, OsBAT1, OsPUT1, and HsSLC7A7 among the annotated/known proteins. These motifs searched in the Saccharomyces cerevisiae protein database identified proteins of diverse family and function, however, motif 1 was common in transporter proteins such as 
ScMUP3, ScAGP2, ScLYP1, ScAGP1, ScDIP5, ScALP1, ScCAN1, ScBAP3, ScTAT1, ScMUP1, ScGNP1, ScHNM1, ScAGP3, ScSSY1, ScTPO1, ScTPO5, ScGAP1, ScHIP1 all of which are associated with amino acid and/or polyamine transport. When OsLATs were excluded in the motif search, motifs identified (supplementary figure S3) were different from what is shown in Fig. 1c. The new motif 1, when searched in the Arabidopsis protein database, was also found to be conserved in AtCAT1-9, AtBAT1, AtAAP2, 4, and 6.

A FWC signature motif in the $\mathrm{PM}_{\text {asseq }}$ domain at the C-termini, conserved in yeast amino acid permeases, that is responsible for plasma membrane association in amino acid transporters localized in the plasma membrane (Popov-Celeketic et al. 2016) was absent in all five LAT proteins.

Loss-of-function of PAR1/LAT4 causes increased sensitivity to exogenous leucine In our growth assays, parl-5 seedlings were visibly smaller than the corresponding WT seedlings in MS medium that contained nitrate, and in peatbased growth medium that contained both organic and inorganic forms of nitrogen (supplementary figure S1c-d). However, growth studies with branchedchain amino acids (BCAAs) such as leucine, isoleucine, or valine as sole source of nitrogen did not support sufficient growth, to allow a comparison between the two genotypes. Thus, we used gradually increasing concentration of leucine at $0 \mathrm{mM}, 1 \mathrm{mM}$, and $2 \mathrm{mM}$ with $3 \mathrm{mM}$ nitrate in the MS-agar medium and observed growth of par1-5 in comparison with WT. Mutant par1-5 seedlings produced $55 \%$ biomass compared to WT on MS medium containing $3 \mathrm{mM}$ nitrate and $0 \mathrm{mM}$ leucine (Fig. 2a, b). In the presence of $1 \mathrm{mM}$ leucine in the growth medium, par1- 
5 seedlings produced $48 \%$ of the biomass compared to the corresponding WT seedlings (Fig. 2b). In the presence of $2 \mathrm{mM}$ leucine, par1-5 seeds had low germination rates compared to the corresponding WT seedlings. In the presence of $2 \mathrm{mM}$ leucine, parl-5 seedlings showed a significant $98 \%$ reduction in biomass compared to WT on the same plate.

PAR1/LAT4 transcripts are present in all organs in maturing plants

Analyzing the PAR1/LAT4 expression using the public database Arabidopsis eFP Browser (At-TAX) showed PAR1/LAT4 mRNA in leaf, stem, flower, silique, whole seedling, and shoot apex. At a tissue-specific level, the Arabidopsis eFP Browser showed PARI/LAT4 expression in the leaf mesophyll and guard cells, stem epidermis, and fruit carpels (http://bbc.botany.utoronto.ca/efp/). Li et al. (2013) demonstrated ubiquitous expression of PAR1/LAT4 throughout Arabidopsis development. We conducted an organ-specific quantitative RT-PCR to see the PAR1/LAT4 transcript level in various organs. The PAR1/LAT4 transcripts were detected in all organs with a higher abundance in the rosette leaves (Fig. 3).

Dark treatment causes leucine accumulation in par1-5

A radio labeled leucine uptake study showed no variation in leucine uptake between par1-5 and WT (Fig. 4a, b). Studies were repeated with seedlings treated in the dark. When seedlings were grown in the dark for 27 hours prior to the isotopic feeding, parl-5 seedlings accumulated significantly more leucine as measured by the ${ }^{14} \mathrm{C}$ - and ${ }^{3} \mathrm{H}$ - accumulation, compared to WT (Fig. 4c, d). Increased accumulation of leucine by par1-5 was also observed in leaf protoplasts 
of dark-treated plants. The average diameter of leaf protoplasts in WT was 0.51 $\mu \mathrm{m}$, while it was $0.38 \mu \mathrm{m}$ in par1-5, a $25 \%$ decrease (Fig. 4e). However, WT protoplasts accumulated $43.5 \mathrm{pmol} .10^{5} \mathrm{cells}^{-1} \cdot \mathrm{hr}^{-1}{ }^{14} \mathrm{C}$-leucine, whereas parl-5 protoplasts accumulated $52.7 \mathrm{pmol} \cdot 10^{5} \mathrm{cells}^{-1} \cdot \mathrm{hr}^{-1}$, a $21 \%$ increase (Fig. 4f).

PAR1/LAT4 shows a cumulative growth reduction in the absence of sucrose

To test whether the reduced availability of sucrose could affect par1-5 growth, an assay was used with the level of nitrogen kept constant but sucrose supplement altered. In the presence of $1 \%$ sucrose in the growth medium, par1-5 showed $32 \%$ and $12 \%$ reduction in total biomass and root length, respectively, compared to the corresponding WT (Fig. 5). This was consistent with previous results that showed reduced biomass of par1-5 compared to WT in MS medium and in soil (supplementary figure. S1, Fig. 2). Without sucrose in the growth medium, reductions in biomass and root length in par $1-5$ were $51 \%$ and $52 \%$, respectively, compared to the corresponding WT (Fig. 5a-c). In the absence of sucrose, par1-5 showed a significant $54 \%$ and $57 \%$ reduction in biomass and root length, respectively, compared to par 1-5 growth with sucrose while no significant growth reduction was observed in WT grown with or without sucrose (Fig. 5a-c).

In the absence of sucrose, par1-5 accumulates free amino acids in the shoots

Free amino acids were analyzed in the roots and shoots of WT and par1-5 seedlings, grown with or without sucrose in the growth medium (Fig. 5a). On medium without sucrose, the total free amino acid content in parl-5 seedlings was $176 \%$ higher in the shoots and $80 \%$ lower in the roots compared to WT plants (Table 1). With sucrose in the growth medium, parl-5 showed no significant 
difference in the total free amino acid content in the roots and shoots compared to that in the corresponding WT (Table 1). The addition of sucrose increased the amino acid content in WT shoots by $67 \%$ and decreased it in parl-5 shoots by $41 \%$ compared to the same genotype grown without sucrose. In roots, addition of sucrose caused an increase in the free amino acid content in WT and parl-5 by $135 \%$ and $809 \%$, respectively, compared to that in the same genotype grown in the absence of sucrose (Table 1). In the absence of sucrose in the growth medium, where the loss of PAR1/LAT4 function was evident by reduced biomass (Fig. 5ac), parl-5 accumulated free amino acids in the shoots (Table 1). The accumulation and distribution of free amino acids in par1-5 was significantly altered compared to WT when sucrose supplement was altered in the growth medium.

\section{Discussion}

PAR1/LAT4-family transporters interact with a broad range of substrates

Phylogenetically, PAR1/LAT4 belongs to the L-type Amino acid Transporter (LAT) family and/or Polyamine H-Symporter (PHS) family (Okumoto and Pilot 2011; Wipf et al. 2002). PAR1/LAT4-family proteins conserve domains and motifs that are common in known amino acid and/or polyamine transporters (Fig. 1b-c). The predicted biological process and molecular function for all five PAR1/LAT4-family members were amino acid transport and amino acid transport activity (supplementary table $\mathrm{S} 1$ ). While in-silico characterization provided a strong suggestion that PAR1/LAT4-family has a role in amino acid transport, experimental evidences suggest that these transporters interact with a broad range 
of substrates. Loss of PAR1/LAT4 function showed increased paraquat tolerance compared to WT suggesting that PAR1/LAT4 mediates paraquat transport (Dong et al. 2016; Li et al. 2013). Another member of this family, RMV1/LAT1 mediated increased polyamine uptake when over-expressed and increased paraquat tolerance when knocked out (Fujita et al. 2012), showed increased cadaverine sensitivity when over-expressed (Sagor et al. 2016) and mediated thiamine transport (Martinis et al. 2016). In this report we have demonstrated that PAR1/LAT4 plays a role in leucine transport. We investigated the role of PAR1/LAT4 in leucine transport by growing par1-5 on MS medium containing nitrate and leucine, as well as, by conducting radio-labelled leucine uptake studies. We were particularly interested to investigate if par1-5 shows variation in leucine uptake because PAR1 was initially classified as LAT4 and LATs in animal have higher affinity for BCAAs (Mastroberardino et al. 1998; Verrey 2003). Moreover, BCAAs were previously shown to produce significant growth inhibition when used as a source of nitrogen, making them a good substrate to study mutants for amino acid transporters (Chen et al. 2010; Forsum et al. 2008; Miflin 1969; Miflin and Cave 1972). We conducted studies with gradually increasing concentration of leucine with $3 \mathrm{mM}$ nitrate supplement to investigate growth variation in par1-5 compared to WT. Leucine was used as a representative of the BCAA group, and $3 \mathrm{mM}$ nitrate was used because this concentration was sufficient to support seedling growth on plates for a period of up to four weeks. Mutant par1-5 seedlings were hypersensitive to the presence of leucine in the growth medium (Fig. 2). In radio-labelled leucine uptake studies, par1-5 showed 
increased accumulation of leucine in leaf mesophyll cell protoplasts and in seedlings upon dark treatment (Fig. 4c, d, f). Our studies along with published reports indicate that PAR1/LAT4-family transporters have a broad substrate specificity including amino acid leucine, polyamine, paraquat, cadaverine, and thiamine. Amino acid transporters in both plant and animal systems, even when specific to only amino acids, are broadly specific, although they have a higher or lower affinity for certain types of amino acids such as acidic, basic, or neutral.

\section{PAR1/LAT4 harbors a complex transport mechanism}

LATs in animals exchange intra-cellular amino acids for extra-cellular amino acids at a 1:1 stoichiometry (Verrey 2003). Animal LATs require binding with a heavy-chain glycoprotein subunit to become functional (Mastroberardino et al. 1998; Pfeiffer et al. 1999; Rossier et al. 1999). Although Arabidopsis PAR1/LAT4-family proteins share low $(\sim 20 \%)$ sequence similarity with animal LATs (Fujita and Shinozaki 2014), the possibility that PAR1/LAT4 and members of its family interact with other subunits to localize and function properly remains to be tested. While RMV1/LAT1 was confirmed to be localized to the plasma membrane (Fujita et al. 2012), when GFP was fused to the c-terminus of PAR1/LAT4 and LAT3, they were localized to the Golgi apparatus and endoplasmic reticulum, respectively ( $\mathrm{Li}$ et al. 2013). Knocking out and overexpressing $R M V 1 / L A T 1$ in Arabidopsis caused reduced, and increased paraquat uptake, respectively, compared to the control. However, in the polyamine uptake studies, $R M V 1 / L A T 1$ over-expression showed increased uptake while the mutant showed no difference compared to the control (Fujita et al. 2012). In studies with 
PAR1/LAT4, the parl mutant showed reduced accumulation of paraquat in the chloroplast but no difference in paraquat uptake compared to the control (Li et al. 2013). In this report, the PAR1/LAT4 affected leucine transport in seedlings and leaf mesophyll cells when plants were treated in the dark (Fig. 4c, d, f). Dong et al (2016) demonstrated that a PAR1/LAT4 mutant's (pqr2) ability to provide paraquat tolerance is compromised when co-treated with paraquat and abscisic acid (Dong et al. 2016). While mutant parl-5 showed reduced paraquat accumulation, it showed increased leucine accumulation, an opposite transport direction for these two substrates.

\section{PAR1/LAT4 transports leucine upon dark induction}

In our studies PAR1/LAT4 facilitated leucine transport in vivo. This ability was detected when plants were exposed to a prolonged dark period. Dark treatment was used to induce expression of the PAR1/LAT4 gene in WT seedlings because microarray studies indicated that PAR1/LAT4 transcript expression is highly induced under reduced light intensity or in continuous dark (Covington and Harmer 2007; Edwards and Millar 2007; Michael et al. 2008). Compared to WT, par1-5 seedlings and leaf protoplasts showed increased accumulation of ${ }^{14} \mathrm{C}$ leucine and ${ }^{3} \mathrm{H}$-leucine (Fig. 4c, d, and f). Although, dark treatment was primarily used to induce PARI/LAT4 transcript level, and it caused significantly increased accumulation of leucine or its metabolic product in the parl-5 seedlings and protoplast, subsequent expression analysis showed ubiquitous and constitutive expression of PAR1/LAT4 in all organs throughout Arabidopsis development (Fig.

3) (Li et al. 2013). Increased accumulation of leucine in par1-5 seedlings upon 
dark treatment was more likely due to factors (triggered by dark treatment) that influenced the transport activity of PAR1/LAT4 rather than producing increased number of transcript. Thus, we considered testing other factors such as sucrose level that are altered during the dark cycle in plants. We have demonstrated that lack of sucrose in the growth medium causes a biomass reduction in par1-5 seedlings (Fig. 5a-c), and significantly alters the accumulation and distribution of free amino acids in par1-5 compared to WT (Table 1). These studies suggest that PAR1/LAT4 plays a role in transporting leucine, and this activity is influenced by dark cycle- derived physiological conditions such as low sucrose concentration.

\section{Conclusion}

This report showed PAR1/LAT4's ability to facilitate leucine transport and previous reports demonstrated its ability to import paraquat into chloroplast (Li et al. 2013). Depending on the substrate type and physiological conditions, PAR1/LAT4 showed transport characteristics which are similar to an antiporter.

\section{Acknowledgments}

This research was funded by Natural Sciences and Engineering Research Council of Canada (NSERC) and Alberta Crop Industry Development Fund (ACIDF) to AGG.

\section{References}


Alonso, J.M., Stepanova, A.N., Leisse, T.J., Kim, C.J., Chen, H., Shinn, P., et al. 2003. Genome-wide insertional mutagenesis of Arabidopsis thaliana. Science, 301(5633): 653-657. doi:10.1126/science.1086391

Altschul, S.F., Madden, T.L., Schaffer, A.A., Zhang, J., Zhang, Z., Miller, W., et al. 1997. Gapped BLAST and PSI-BLAST: a new generation of protein database search programs. Nucleic Acids Res. 25(17): 3389-3402. PMCID:146917

Bailey, T. and Elkan, C. (1994). Fitting a mixture model by expectation maximization to discover motifs in biopolymers. Menlo Park, California: AAAI Press, .

Bailey, T.L. and Gribskov, M. 1998. Combining evidence using p-values: application to sequence homology searches. Bioinformatics, 14(1): 48-54. doi:10.1093/bioinformatics/14.1.48

Chen, H., Saksa, K., Zhao, F., Qiu, J., and Xiong, L. 2010. Genetic analysis of pathway regulation for enhancing branched-chain amino acid biosynthesis in plants. Plant J. 63(4): 573-583. doi:10.1111/j.1365-313X.2010.04261.X

Covington, M.F. and Harmer, S.L. 2007. The circadian clock regulates auxin signaling and responses in Arabidopsis. PLoS Biol. 5(8): e222. doi:10.1371/journal.pbio.0050222

Dong, S., Hu, H., Wang, Y., Xu, Z., Zha, Y., Cai, X., et al. 2016. A pqr2 mutant encodes a defective polyamine transporter and is negatively affected by 
ABA for paraquat resistance in Arabidopsis thaliana. J. Plant Res. 129(5): 899-907. doi:10.1007/s10265-016-0819-y

Edwards, K.D. and Millar, A.J. 2007. Analysis of circadian leaf movement rhythms in Arabidopsis thaliana. Methods Mol.Biol. 362:103-113. $10.1007 / 978-1-59745-257-1 \_7$

Forsum, O., Svennerstam, H., Ganeteg, U., and Nasholm, T. 2008. Capacities and constraints of amino acid utilization in Arabidopsis. New Phytol. 179(4): 1058-1069. doi:10.1111/j.1469-8137.2008.02546.x

Fujita, M. and Shinozaki, K. 2014. Identification of polyamine transporters in plants: paraquat transport provides crucial clues. Plant Cell Physiol. 55(5): 855-861. doi:10.1093/pcp/pcu032

Fujita, M., Fujita, Y., Iuchi, S., Yamada, K., Kobayashi, Y., Urano, K., et al. 2012. Natural variation in a polyamine transporter determines paraquat tolerance in Arabidopsis. Proc. Natl. Acad. Sci. U S A 109(16): 63436347. doi:10.1073/pnas.1121406109

Kaundal, R., Saini, R., and Zhao, P.X. 2010. Combining machine learning and homology-based approaches to accurately predict subcellular localization in Arabidopsis. Plant Physiol. 154(1): 36-54. doi:10.1104/pp.110.156851

Lam, H.M., Coschigano, K.T., Oliveira, I.C., Melo-Oliveira, R., and Coruzzi, G.M. 1996. The Molecular-Genetics of Nitrogen Assimilation into Amino Acids in Higher Plants. Annu. Rev. Plant Physiol. Plant Mol. Biol. 47(1): 569-593. doi:10.1146/annurev.arplant.47.1.569 
Li, J., Mu, J., Bai, J., Fu, F., Zou, T., An, F., et al. 2013. Paraquat Resistant1, a Golgi-localized putative transporter protein, is involved in intracellular transport of paraquat. Plant Physiol. 162(1): 470-483. doi:10.1104/pp.113.213892

Martinis, J., Gas-Pascual, E., Szydlowski, N., Crevecoeur, M., Gisler, A., Burkle, L., et al. 2016. Long-Distance Transport of Thiamine (Vitamin B1) Is Concomitant with That of Polyamines. Plant Physiol. 171(1): 542-553. doi:10.1104/pp.16.00009

Mastroberardino, L., Spindler, B., Pfeiffer, R., Skelly, P.J., Loffing, J., Shoemaker, C.B., et al. 1998. Amino-acid transport by heterodimers of 4F2hc/CD98 and members of a permease family. Nature, 395(6699): 288291. doi:10.1038/26246

Michael, T.P., Mockler, T.C., Breton, G., McEntee, C., Byer, A., Trout, J.D., et al. 2008. Network discovery pipeline elucidates conserved time-of-dayspecific cis-regulatory modules. PLoS Genet. 4(2): e14. doi:10.1371/journal.pgen.0040014

Miflin, B.J. 1969. The Inhibitory Effects of Various Amino Acids on the Growth of Barley Seedlings. J. Exp. Bot. 20(4): 810-819.

Miflin, B.J. and Cave, P.R. 1972. The Control of Leucine, Isoleucine, and Valine Biosynthesis in a Range of Higher Plants. J. Exp. Bot. 23(2): 511-516. doi: $10.1093 / \mathrm{jxb} / 23.2 .511$ 
Mulangi, V., Chibucos, M.C., Phuntumart, V., and Morris, P.F. 2012. Kinetic and phylogenetic analysis of plant polyamine uptake transporters. Planta, 236(4): 1261-1273. doi:10.1007/s00425-012-1668-0

Murashige, T. and Skoog, F. 1962. A revised medium for rapid growth and bioassays with tobacco tissue cultures. Physiol. Plant, 15(473 - 497.

Nasholm, T., Kielland, K., and Ganeteg, U. 2009. Uptake of organic nitrogen by plants. New Phytol. 182(1): 31-48. doi:10.1111/j.1469-8137.2008.02751.x

Okumoto, S. and Pilot, G. 2011. Amino acid export in plants: a missing link in nitrogen cycling. Mol. Plant, 4(3): 453-463. doi:10.1093/mp/ssr003

Pfeiffer, R., Rossier, G., Spindler, B., Meier, C., Kuhn, L., and Verrey, F. 1999. Amino acid transport of $\mathrm{y}+\mathrm{L}-\mathrm{type}$ by heterodimers of $4 \mathrm{~F} 2 \mathrm{hc} / \mathrm{CD} 98$ and members of the glycoprotein-associated amino acid transporter family. EMBO J. 18(1): 49-57. doi:10.1093/emboj/18.1.49

Popov-Celeketic, D., Bianchi, F., Ruiz, S.J., Meutiawati, F., and Poolman, B. 2016. A Plasma Membrane Association Module in Yeast Amino Acid Transporters. J. Biol. Chem. 291(31): 16024-16037. doi:10.1074/jbc.M115.706770

Pratelli, R., Voll, L.M., Horst, R.J., Frommer, W.B., and Pilot, G. 2010. Stimulation of nonselective amino acid export by glutamine dumper proteins. Plant Physiol. 152(2): 762-773. doi:10.1104/pp.109.151746 
Rentsch, D., Schmidt, S., and Tegeder, M. 2007. Transporters for uptake and allocation of organic nitrogen compounds in plants. FEBS Lett. 581(12): 2281-2289. doi:10.1016/j.febslet.2007.04.013

Rossier, G., Meier, C., Bauch, C., Summa, V., Sordat, B., Verrey, F., et al. 1999. LAT2, a new basolateral 4F2hc/CD98-associated amino acid transporter of kidney and intestine. J. Biol. Chem. 274(49): 34948-34954.

Sagor, G.H., Berberich, T., Kojima, S., Niitsu, M., and Kusano, T. 2016. Spermine modulates the expression of two probable polyamine transporter genes and determines growth responses to cadaverine in Arabidopsis. Plant Cell Rep. 35(6): 1247-1257. doi:10.1007/s00299-016-1957-3

Tegeder, M. 2012. Transporters for amino acids in plant cells: some functions and many unknowns. Curr. Opin. Plant Biol. 15(3): 315-321. doi:10.1016/j.pbi.2012.02.001

Tuin, L.G. and Shelp, B.J. 1994. In situ [14C]Glutamate Metabolism by Developing Soybean Cotyledons I. Metabolic Routes. J. Plant Physiol. 143(1): 1-7. doi:10.1016/s0176-1617(11)82089-4

Verrey, F. 2003. System L: heteromeric exchangers of large, neutral amino acids involved in directional transport. Pflugers Arch. 445(5): 529-533. doi:10.1007/s00424-002-0973-z

Wipf, D., Ludewig, U., Tegeder, M., Rentsch, D., Koch, W., and Frommer, W.B. 2002. Conservation of amino acid transporters in fungi, plants and animals. Trends Biochem. Sci. 27(3): 139-147. 
Woodward, C., Henderson, J.J.W., and Wielgos, T. 2007. High-speed amino acid analysis (AAA) on $1.8 \mu \mathrm{m}$ reversed-phase (RP) columns. Wilmington, DE, USA: Agilent Technologies

Yoo, S.D., Cho, Y.H., and Sheen, J. 2007. Arabidopsis mesophyll protoplasts: a versatile cell system for transient gene expression analysis. Nat. Protoc. 2(7): 1565-1572. doi:10.1038/nprot.2007.199 
Table 1. Free amino acid analysis in the roots and shoots of parl-5 seedlings in comparison with WT grown in the presence or absence of $1 \%$ sucrose in the growth medium.

\begin{tabular}{|c|c|c|c|c|c|c|c|c|c|c|c|c|}
\hline \multirow[b]{3}{*}{ Free AA } & \multicolumn{6}{|c|}{$1 \%$ sucrose } & \multicolumn{6}{|c|}{$0 \%$ sucrose } \\
\hline & \multicolumn{3}{|c|}{ Shoot (nmol.g-1 FW) } & \multicolumn{3}{|c|}{ Root (nmol.2seedling $\left.{ }^{-1}\right)$} & \multicolumn{3}{|c|}{ Shoot(nmol.g $\left.{ }^{-1} \mathrm{FW}\right)$} & \multicolumn{3}{|c|}{ Root (nmol.2seedling ${ }^{-1}$ ) } \\
\hline & WT & parl-5 & $\%$ Change & $\begin{array}{l}\text { WT } \\
\end{array}$ & parl-5 & \% Change & WT & parl-5 & $\%$ Change & WT & parl-5 & $\%$ Change \\
\hline Asp & 1635.98 & 2369.75 & 44.85 & 9.67 & 8.72 & -9.90 & 1230.56 & 934.05 & -24.10 & 4.13 & 0.22 & -94.72 \\
\hline Glu & 4086.46 & 3753.03 & -8.16 & 32.20 & 26.89 & -16.47 & 2252.21 & 3107.20 & +37.96 & 11.78 & 0.45 & -96.16 \\
\hline Asn & 247.24 & 243.41 & -1.55 & 7.40 & 5.42 & -26.71 & 138.43 & 322.83 & +133.20 & 2.60 & 0.28 & -89.18 \\
\hline Ser & 1842.05 & 1379.72 & -25.10 & 18.98 & 12.85 & -32.32 & 581.71 & 3187.72 & $+447.99 *$ & 5.26 & 0.96 & -81.67 \\
\hline Gln & 2126.76 & 1739.75 & -18.20 & 45.52 & 32.53 & -28.55 & 722.59 & 1268.02 & +75.48 & 15.65 & 1.46 & -90.66 \\
\hline His & 39.59 & 37.63 & -4.96 & 0.90 & 0.72 & -20.13 & 32.67 & 68.49 & +109.65 & 0.39 & 0.04 & $-90.46^{*}$ \\
\hline Gly & 242.60 & 124.99 & -48.48 & 2.74 & 1.98 & -27.64 & 55.70 & 900.14 & +1516.10 & 1.61 & 0.80 & -50.07 \\
\hline Thr & 583.26 & 466.22 & -20.07 & 8.63 & 6.06 & -29.84 & 192.27 & 825.91 & $+329.56^{*}$ & 2.81 & 0.35 & -87.68 \\
\hline Arg & 42.42 & 46.74 & 10.18 & 1.01 & 0.77 & -23.49 & 41.87 & 134.07 & $+220.18^{*}$ & 0.53 & 0.13 & -75.85 \\
\hline Ala & 497.98 & 442.68 & -11.10 & 5.13 & 3.82 & -25.54 & 256.64 & 902.32 & $+251.58^{*}$ & 2.45 & 0.49 & -79.84 \\
\hline Tyr & 28.40 & 34.98 & 23.15 & 0.71 & 0.59 & -16.07 & 43.37 & 225.40 & $+419.69^{*}$ & 0.45 & 0.24 & $-46.84^{*}$ \\
\hline Cys & 4.81 & 10.45 & 117.23 & 0.19 & 0.15 & -21.39 & 17.43 & 160.11 & $+818.34 * *$ & 0.21 & 0.15 & -25.69 \\
\hline Val & 1785.28 & 2201.67 & 23.32 & 42.28 & 33.96 & -19.69 & 2119.88 & 8235.10 & $+288.47 *$ & 25.66 & 8.11 & -68.40 \\
\hline Met & 22.81 & 28.10 & 23.21 & 0.43 & 0.34 & -22.37 & 34.82 & 131.32 & $+277.13^{*}$ & 0.27 & 0.12 & $-56.36^{*}$ \\
\hline Trp & 15.80 & 19.46 & 23.13 & 0.24 & 0.21 & -10.52 & 20.89 & 97.51 & $+366.83 *$ & 0.20 & 0.08 & $-57.50^{*}$ \\
\hline Phe & 39.98 & 36.13 & -9.63 & 0.58 & 0.53 & -8.44 & 42.17 & 247.73 & $+487.52 * *$ & 0.34 & 0.19 & -44.75 \\
\hline Ile & 50.29 & 54.91 & 9.19 & 1.55 & 1.21 & -22.08 & 56.07 & 301.07 & $+436.94 *$ & 0.84 & 0.29 & $-65.79^{*}$ \\
\hline Leu & 48.85 & 57.11 & 16.92 & 2.10 & 1.77 & -15.87 & 52.71 & 294.73 & $+459.19^{*}$ & 1.09 & 0.25 & -77.36 \\
\hline Lys & 26.16 & 39.96 & 52.77 & 0.81 & 0.67 & -16.58 & 121.68 & 773.71 & +535.84 & 0.85 & 0.71 & -16.49 \\
\hline Total & 13366.72 & 13087 & -2.09 & 181.08 & 139.19 & -23.13 & 8013.68 & 22117.43 & $176.00^{*}$ & 77.11 & 15.32 & $-80.13^{*}$ \\
\hline
\end{tabular}

*, Pvalue $<0.05$; **, Pvalue $<0.01$;**, Pvalue $<0.001$. Each data is an average of three biological replications with two seedlings per replication. 


\section{Botany}

Page 28 of 37

https://mc06.manuscriptcentral.com/botany-pubs 


\section{Figure legends}

Fig. 1. In-silico characterization of the Arabidopsis PAR1/LAT4-family proteins.

a. A cartoon representation showing the 10 transmembrane domains in PAR1/LAT4 protein with both $\mathrm{C}$ - and N-termini in the cytoplasmic side.

b. Amino acid permease domain PF13520 is conserved in all five members (LAT1-5) of the Arabidopsis PAR1/LAT4-family. Grey bar shows the length of amino acid sequence and red bar shows the position and extent of the domain in each protein.

c. A block diagram of non-overlapping tiling of motifs found common in the Arabidopsis PAR1/LAT4- family and related proteins in Oryza sativa, Homo sapiens, and Mus musculus identified using MEME motif search tool. Each colored box represents a specific motif in the protein. The order of the motifs indicates their position within each protein sequence.

Fig. 2. Mutant par1-5 shows increased sensitivity to exogenous leucine

d. Mutant par1-5 seedlings are more sensitive to exogenous leucine compared to WT. Variable concentrations of L-leucine were added to $0.5 \mathrm{xMS}$ medium with $3 \mathrm{mM}$ nitrate as the source of nitrogen.

e. A quantitative representation shows significantly increased sensitivity of the par1-5 mutant seedlings compared to WT to the presence of variable concentrations of leucine measured by the reduction in fresh-weight of biomass.

The relative percentage of growth was calculated assuming the biomass obtained by the corresponding WT as $100 \%$. Error bar represents standard deviation of 
three to five biological replications with two seedlings per replication. Photos shown in panel A were taken when the seedlings were two-week old. Each growth condition had three or more plate replications. Each growth experiment was repeated more than twice with similar results. *, Pvalue $<0.05 ; * *$, Pvalue $<0.01$; ***, Pvalue $<0.001)$

Fig. 3. Organ specific expression analyses in mature plants through quantitative RT-PCR detects PAR1/LAT4 transcripts in all organs.

The relative quantitation was calculated using an average expression of PAR1/LAT4 in whole plants as a calibrator and EF1 $\alpha$ as an endogenous control. The error bar represents the average difference between the $R Q_{\min }$ and $R Q_{\max }$ of three biological replications each with three technical replications.

Fig. 4. Radio-labeled leucine uptake for two hours shows increased accumulation of L-leucine measured by ${ }^{14} \mathrm{C}$ - and ${ }^{3} \mathrm{H}$ - signal in parl-5 compared to $\mathrm{WT}$ when seedlings were treated in dark for 27 hours prior to isotopic feeding.

a. Seedlings exposed to light for six hours post night-time show no variation in ${ }^{14} \mathrm{C}$ - accumulation between WT and par1-5.

b. Study as in 'a' shows no variation in ${ }^{3} \mathrm{H}$ - accumulation between WT and par1-5.

c. Seedlings, kept in dark for 27 hours prior to isotopic feeding, show significantly increased accumulation of ${ }^{14} \mathrm{C}$ - in parl-5 compared to WT.

d. Study as in 'c' shows significantly increased accumulation of ${ }^{3} \mathrm{H}-$ in parl5 compared to WT. 
e. Diameter measurement shows significantly smaller size of leaf protoplasts in parl-5 compared to WT. $\mathrm{N}=100$.

f. Leaf protoplasts of parl-5 show significantly increased accumulation of ${ }^{14} \mathrm{C}$ - compared to WT. $\mathrm{N}=3$ to 5 vials with $4 \times 10^{5}$ cells in each vial from 10 independent plants.

In studies a-d Error bar represents standard deviation of three biological replications. *, P-value $<0.05 ; * *$, P-value $<0.01 ; * * *$, P-value $<0.001$.

Fig. 5. In the absence of sucrose in the growth medium, parl-5 shows cumulative growth reduction compared to WT

a. Mutant par1-5 shows reduced biomass and root length compared to WT in the presence of $1 \%$ sucrose in the growth medium. In the absence of sucrose, par1-5 seedlings show further reduction in both biomass and root length compared to those in the presence of sucrose.

b. A quantitative representation of the reduction in biomass in par1-5 compared to WT in the presence or absence of sucrose in the growth medium. $\mathrm{N}=$ 4, with two seedlings per replication.

c. A quantitative representation of the reduction in root-length in par1-5 compared to WT in the presence or absence of sucrose in the growth medium. N= 10.

The percentage of root-length or biomass was calculated by assuming the average growth obtained by the corresponding WT as $100 \%$. The root length measurement and plate photos were taken when the seedlings were nine-day old. 
For the dry weight of biomass, two-week old seedlings were dried at $70^{\circ} \mathrm{C}$ for four hours before taking weight. All statistical significance tests were carried out on average value of the biological replications derived from raw data. Error bar represents standard deviation. *, P-value $<0.05$; **, P-value $<0.01$; ***, P-value $<0.001$. 


\section{Page 33 of 37}

Extracellular

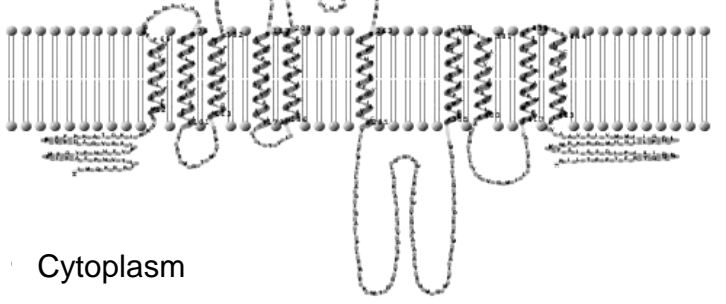

b

\section{Botany}

74aa - 464aa direct

$72 a a-471$ aa direct

LAT2

LAT3

LAT5 52aa - 446aa direct
C

At1G31830/LAT4

OsLAT5/LOC_Os03g37984

At5G05630/LAT1

At1G31820/LAT3

OsLAT1/LOC_Os02947210

At3G19553/LAT5

At3G13620/LAT2

OsLAT4/LOC_Os03g25920

OsLAT2/LOC_Os03g25840

OsLAT3/LOC_Os03g25869

OsLAT7/LOC_Os12939080

MmY+LAT2/NP_848913.1

HsY+LAT2/NP_001070253.1

HsY+LAT1/NP_001119578.1

MmY+LAT1/NP_001240609.1

HsLAT2/AAF20381.1

HsLAT1/BAB70708.1

E.Colitadic

SCHIP1/CAA97217.1

SCGAP1/CAA82113

SCBAP2/CAA85012.1

OsLAT9/LOC_Os08g23440

OsLAT8/LOC_Os01g19850

HsLAT3/NP_001185739.1

At5G49630/AAP6

HsLAT4/DAA05676.1

Fig. 1

$\square$ Motif $1 \square$ Motif $2 \square$ Motif $3 \square$ Motif $4 \square$ Motif $5 \square$ Motif 6

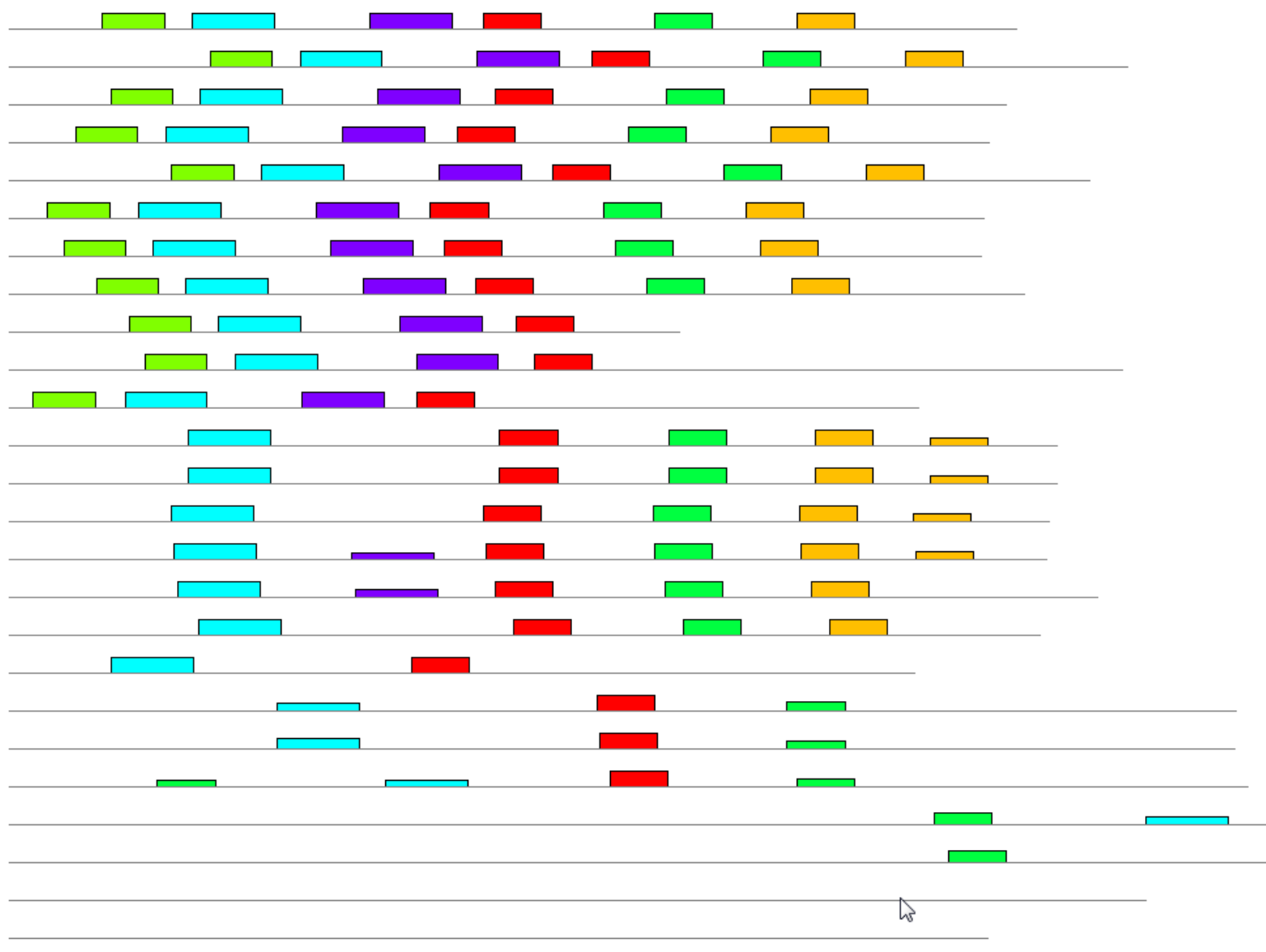

https://mc.06.manuscriptcentral,com/botany-pulbs

600 
a

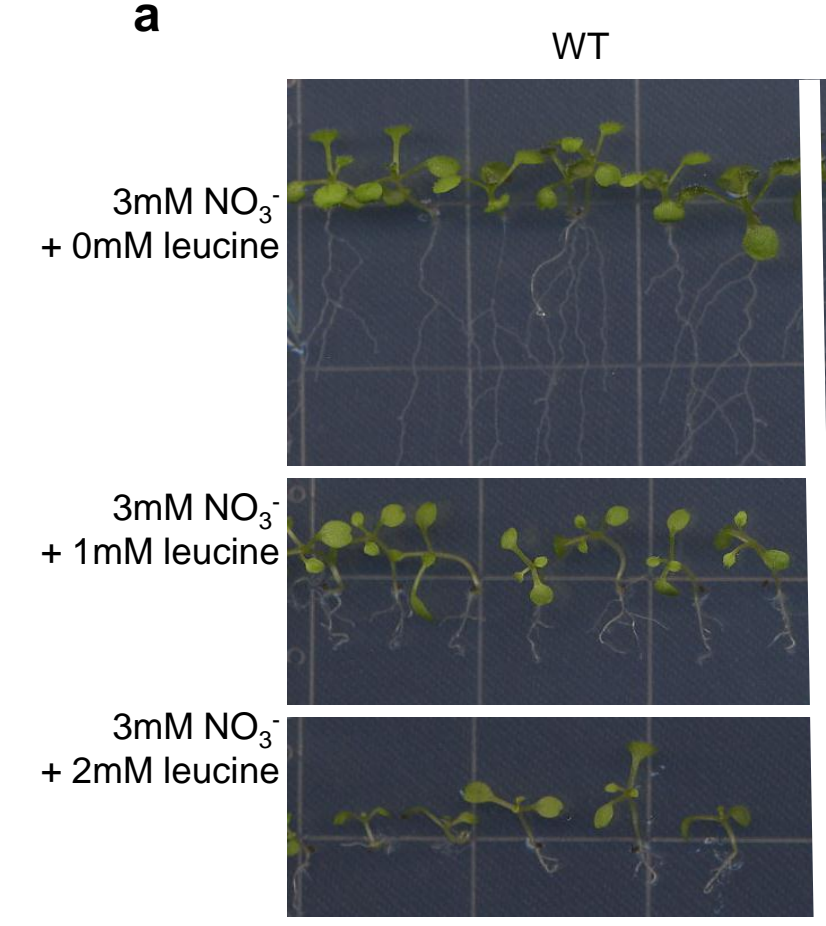

Botany

par1-5

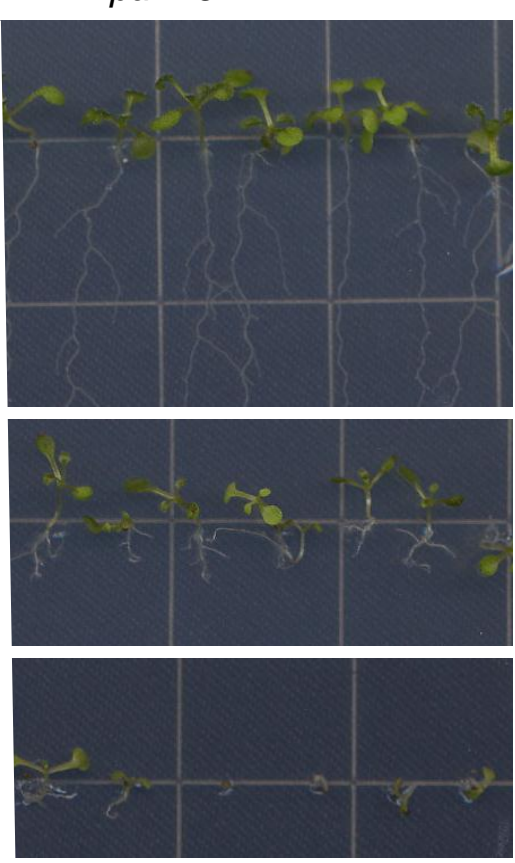

b

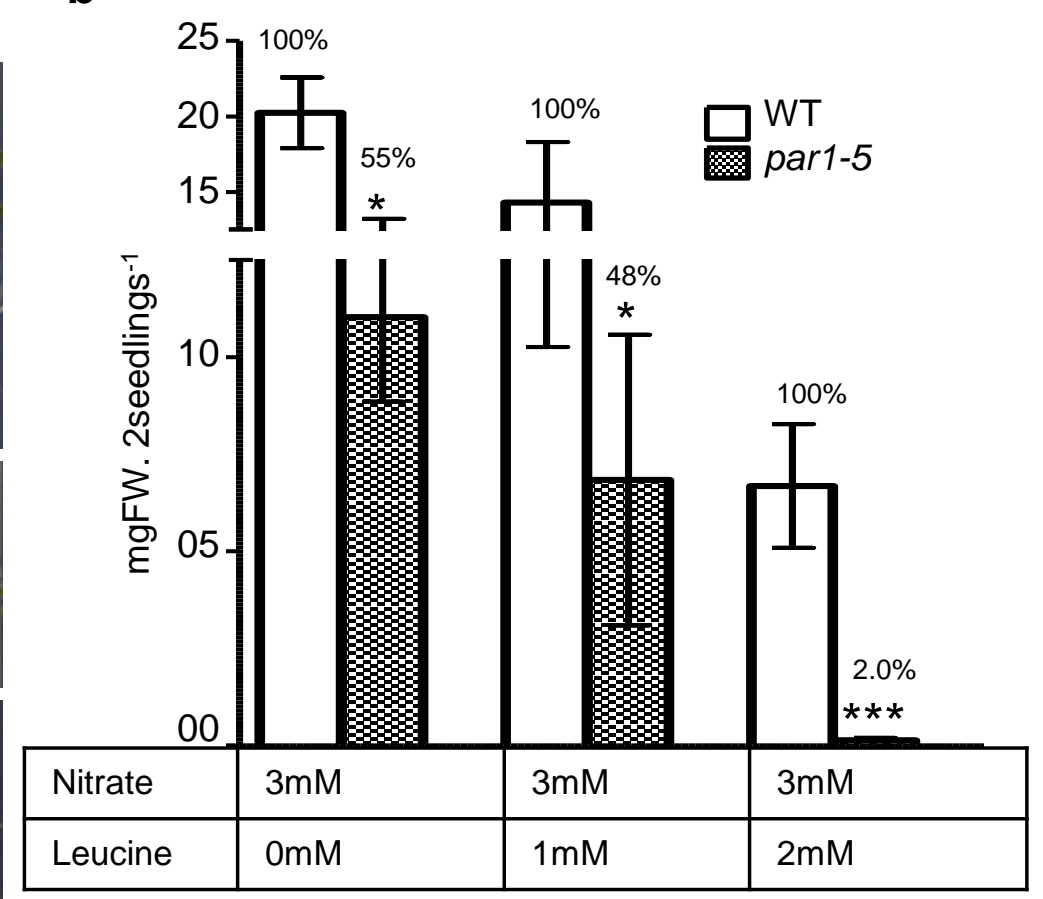

Fig. 2 


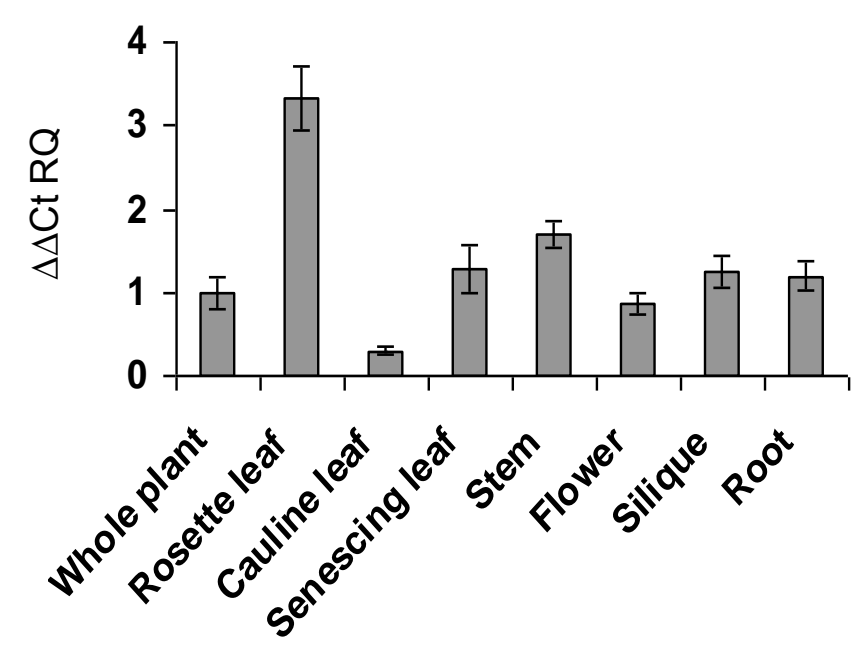

Fig. 3 
$\mathbf{a}$

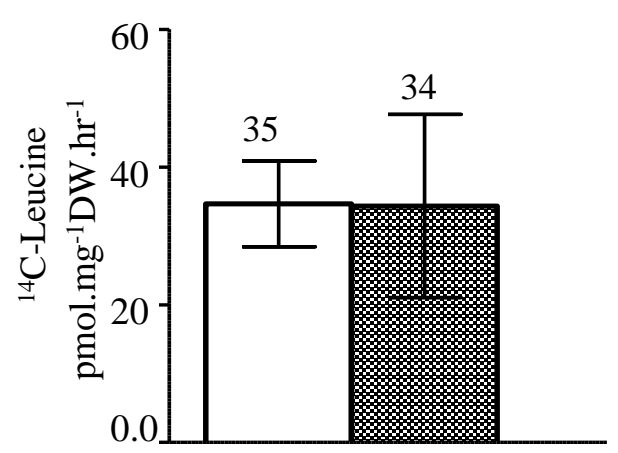

c

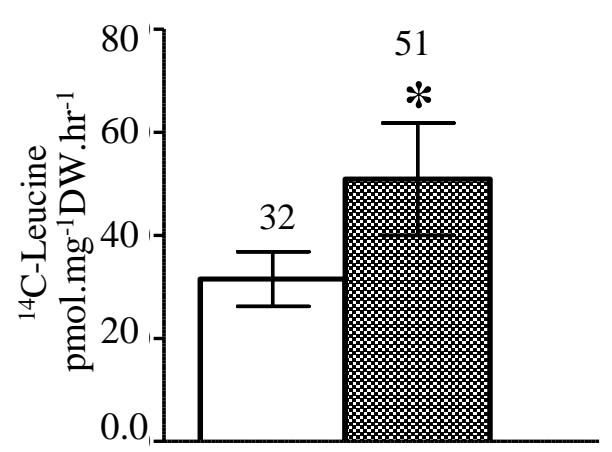

$\mathbf{e}$

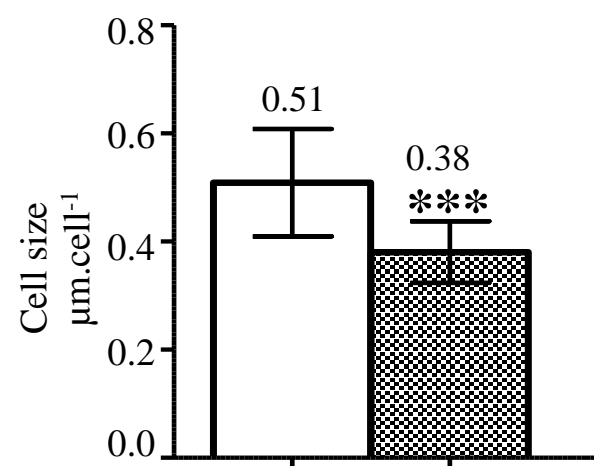

b

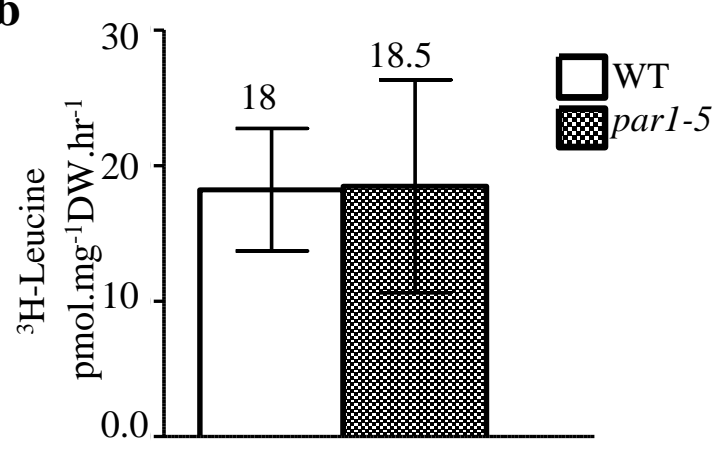

d
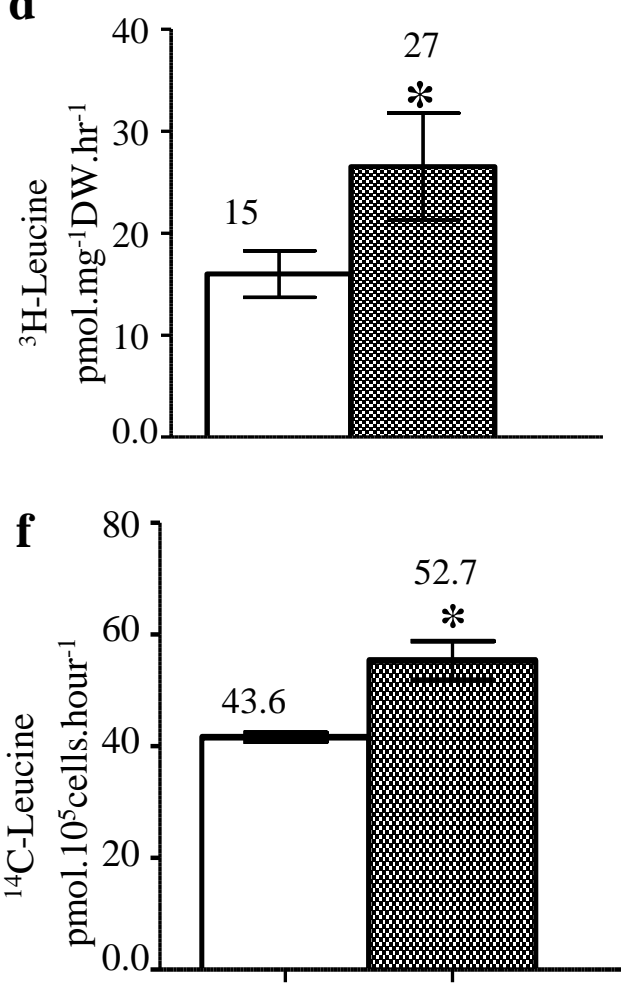

https://mc06.manuscriptcentral.com/botany-pubs

Fig. 4 
a

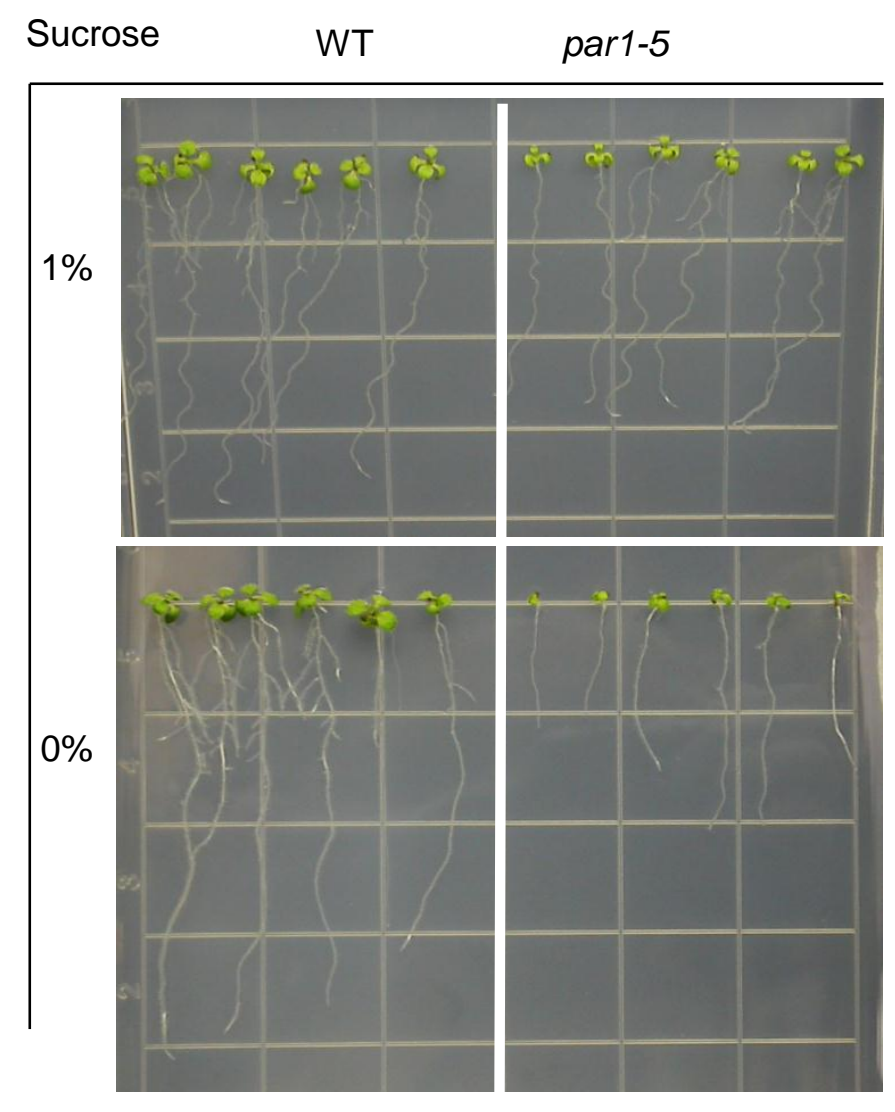

b
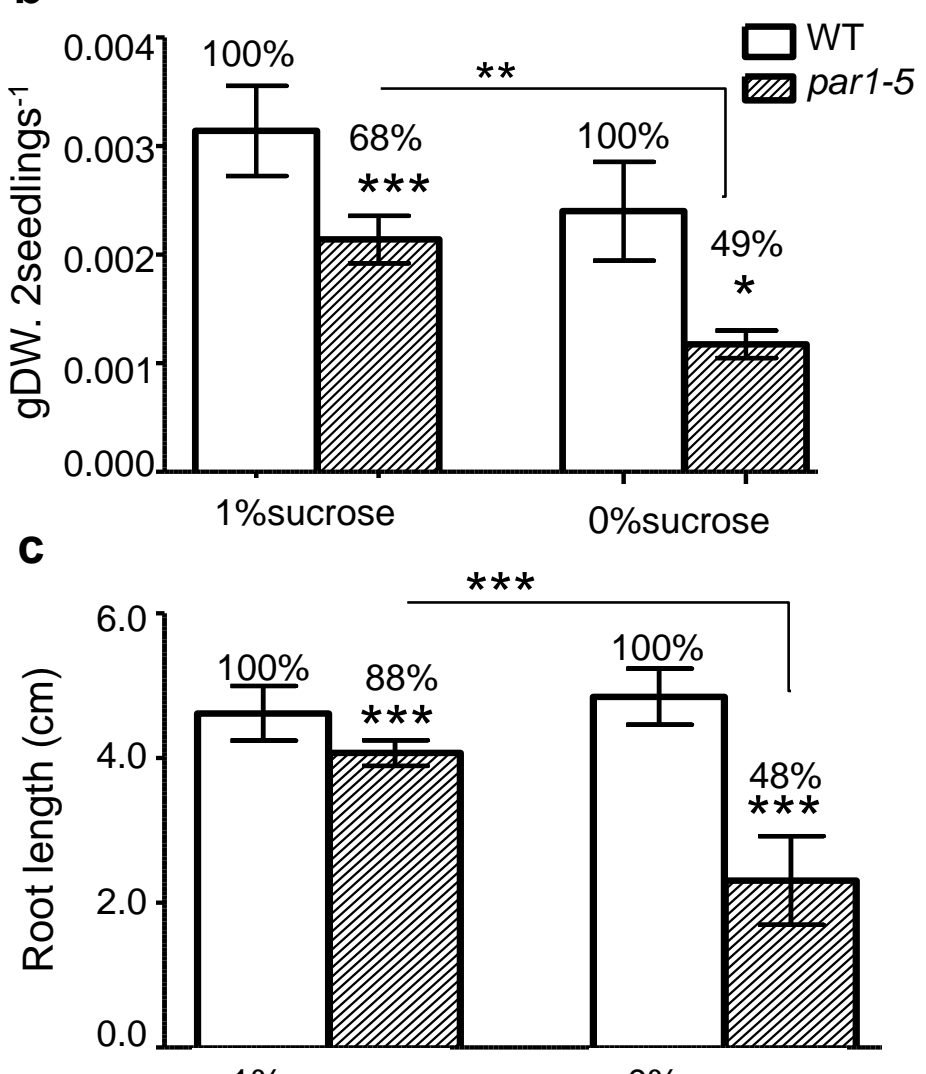

$1 \%$ sucrose
$0 \%$ sucrose

Fig. 5 